\title{
Editorial perspective on Behaviour, Volume 155 (2018)
}

\author{
Brian Wisenden \\ Biosciences Department, Minnesota State University Moorhead, 1104 7th Avenue S, \\ Moorhead, MN, 56563, USA
}

The past 12 months of publications in Behaviour span an interesting range of topics. The paper by Robert Ian Bowers is a fitting place to start because this review provides historical context for the development of Tinbergen's seminal paper in 1963 that defined the "four questions" restated in the frontispiece of this journal. Pre-1963 attempts by Tinbergen to fit behavioural biology into a hierarchical model that linked behaviour with physiology were not successful. These were dropped from the final synthesis articulated in the 1963 paper. Meanwhile insights from Konrad Lorenz and later, a series of developments by Wasserman, Baerends and Hogan led Timberlake \& Grant (1975) to propose the idea of a behavioural system as "a collection of species-typical sensori-motor mechanisms potentially sharing common causal factors". Behavioural systems have increasingly found a home in psychology and learning theory, but less so in ethology. In this review Bowers argues that behavioural systems represent a common foundation for a psychology-ethology integration. Although many interpret Tinbergen's question of 'causation' as encompassing all proximate mechanisms, including psychology, Bowers argues that thinking of behaviour at a system level is useful for ethologists working on questions relevant to psychology, and especially helpful to psychologists trying to integrate their work with ethological literature.

Most papers published in Volume 155 report how behaviour helps animals navigate social and environmental conditions to enhance the probability of survival and ultimately, reproduce. For example, in the past 12 months we have published papers on predator avoidance by cave salamanders, Andean lizards and mountain lizards, Japanese tits and crayfish. We published three papers on vocalizations used by cowbirds, clicking by hippopotamus and 
the effect of age on vocalizations by deer hinds. Consistent inter-individual differences in behaviour, so-called animal "personality", continue to be an active area of research. This year we published six papers on personality in rainbow trout, Trinidadian guppies, the cooperative-nesting cichlid Neolamprologus pulcher, zebra finches, monk parakeets and Floridian songbirds.

The largest classification of papers published this year have been on various aspects of social behaviour. For example, we published papers on mirror self-recognition by cockatoos, claw-waving by hermit crabs, a test of the dear-enemy phenomenon in red squirrels, the role of play bows in canids, social interactions among mountain goats, pupfish, clonal fish, chimpanzees and invasive red-billed leiothrix. There were also some eye-brow-raising studies on strategic pooping by hedgehogs and mobile-phone carrying in humans as a signal of status and social connectiveness.

Among other things, we report in this volume the first intensive study of courtship and mating behaviour in platypuses! Also, Neotropical armoured harvestmen use vision to orient to their eggs, African grey parrots use trial-and-error learning and Goffin's cockatoos can prospectively, but not retrospectively, select functional tools to complete a task. Are insectivores attracted to herbivore-induced plant volatiles? Read the study by Saavedra \& Amo to find out the answer!

The effect of development on the expression of behaviour is another one of Tinbergen's questions. In this volume we report the effect of early life experience on dog behaviour, copulatory rates of seed beetles, HPA-associated genes in the brains of mice, vigilance in chicks and the onset of adult behaviours in dickcissels.

William G. Eberhard wrote an excellent paper on the evolution of modular pattern of webs derived from orbs. Despite "modest neural equipment" orb spiders construct complex webs. By summarizing published data on 15 groups from 19 genera in 8 families, Eberhard documented 69 different evolutionary transitions in web design. Summarized in an extensive table, $91 \%$ of web modifications were overtly behavioural in nature, two-thirds of which were clearly homologous to recognizable ancestral web construction behaviours. The remaining $9 \%$ of changes were the result of changes in the proximate stimuli that guide construction behaviour. There were examples of traits being expressed in new contexts, traits being reduced in expression or lost altogether, other traits that were previously lost were recovered, and some traits were novel innovations with no previous homologue. Taken 
together, this is a delightful read that elegantly illustrates the evolution of behaviour through phylogenetic analysis.

In addition to publishing papers submitted ad hoc to the journal, $B e$ haviour also facilitates publication of special issues on specific topics with the assistance of guest editor(s). This year, we published a special issue on Advances in Disease Ecology Through Contact Networks, guest edited by Stephanie S. Godfrey (University of Otago, Dunedin, New Zealand) and Stephan T. Leu (Macquarie University, Sydney, Australia). Contact network models represent a powerful tool for quantifying the degree of social connectiveness among individuals that ultimately manifest in phenomena at the population level. These data have obvious application to the study of social behaviour ranging from dominance hierarchies, privileged access to prime habitat, and dynamics of mating behaviour. In this special issue, contact network data are used to understand the role of social behaviour and animal movement in the spread of parasites and pathogens within a population. Some individuals are more connected than others, which then carry a greater risk of acquiring parasites, and greater risk of becoming "superspreaders". Does infection change an individual's connectiveness through avoidance by conspecifics (reduced connectiveness) or parasite manipulation (increased connectiveness)? Moreover, contact network models allow behaviourists to collect data on multiple species that host a parasite, as well as track multiple parasites that infect each host in complex multi-layered ecological interactions. This special issue includes contributions from Aiello et al. on sex-biased and seasonal changes in disease transmission in desert tortoises, spread of bovine tuberculosis in brushtail possums by Rouco et al., simulations of how grooming behaviour in various group sizes of nonhuman primates might contribute to risk of spread of disease by Sumner et al., the role of parasite manipulation of host behaviour on contact networks by Poulin, applications to wildlife management by Jones et al., multi-layered analysis of risk of disease transmission by Silk et al., and Gilbertson et al. describe how incorporating genomic metrics of pathogens can greatly enhance the resolution of social contact data and disease dynamics in natural populations. This special issue describes an exciting new direction for behavioural research.

As you can see, there is something for everyone in volume 155 of $\mathrm{Be}$ haviour. Papers cover a range of taxa, and a range of questions in behaviour, ranging from a historical perspective to a special issue on an exciting emerging area of research. 